\title{
Modelling of partially wet particles in DEM simulations of a solid mixing process
}

\author{
Steffen Schmelzle *, Ermek Asylbekov, Benjamin Radel, Hermann Nirschl \\ Karlsruhe Institute of Technology, Institute for Mechanical Process Engineering and Mechanics, Strasse am Forum 8, 76131 Karlsruhe, Germany
}

Keywords:

DEM simulation

Liquid bridge

Granular material

Solids mixing

Drop modelling

\begin{abstract}
A B S T R A C T
It is the basic objective of a solid mixing process to avoid any segregation effects. Especially free flowing bulk solids with weak interparticle adhesive forces tend to percolating segregation. Liquid addition, which is common in mixing granulation processes, will usually reduce the particle segregation due to pendular liquid bridge net works. However, liquid addition can result in a change of the segregation mechanism. For example: water may accumulate selectively in one component when hydrophilic and hydrophobic solids need to be mixed. Heteroagglomerates that consist mainly of one solid component may arise and degrade the final product quality. In this work the Discrete Element Method (DEM) is applied to investigate mixing and segregation of moist par ticulate solids in more detail. During particle collision liquid is transferred from one to the other contact partner until the liquid is uniformly distributed in the bulk. The available liquid bridge models assume complete particle wetting or neglect that the mixing components may differ in their contact angle. We developed the liquid contact dispersion model further in order to realize partial particle wetting that includes drop formation on the particle surface. A comparison between the common film model and the proposed model shows an improvement in experimental validation when the partial particle wetting model is included in the DEM simulation. The assess ment of these models is carried out by evaluating mixing efficiency and liquid distribution.
\end{abstract}

\section{Introduction}

In high shear granulation [1] or tablet coating [2], liquid is often atomized through nozzles and sprayed onto dry particles. To protect the gastric mucosa from aggressive drugs or even for a delayed release of active ingredients, the surface of solid pharmaceutical tablets is often coated in mixing drums. The required tablet quality is significantly influenced by the intra and inter uniformity of the coating. Intra uni formity is the variation of the local layer thickness, while inter unifor mity describes the variation of the coating composition between different tablets. Simulations are becoming increasingly important in order to investigate intra and inter uniformity and to optimize coating or mixing processes. Usually, the evaluation of injection and process control is carried out by post processing algorithms based on particle positioning, orientation and residence time within the spray zone $[3,4]$.

In pharmaceutical high shear granulation, a filling material like lac tose and microcrystalline cellulose [5] has to be briefly dry mixed with a low concentrated active ingredient in order to increase the particle or granule size by subsequently adding a binder [6]. This improves the processability, for example the filling of capsules. Not only the granule size distribution, but also the distribution of liquid within the powder

\footnotetext{
* Corresponding author.

E-mail address: steffen.schmelzle@kit.edu (S. Schmelzle).
}

is influenced by the operating parameters of the high shear mixer. The granule size and the liquid distribution depend on the rate of liquid addition, equally does the product movement behavior in the mixing vessel and the size of the spraying zone [7]. In the case of uneven liquid distribution, over or under humidified areas may arise in the mixing components, which in turn may result in broad granule size distribu tions and inhomogeneous agglomerates [8]. In order to improve the uni formity of humidification, formulation or process variables have to be adjusted experimentally. In case of a formulation change the fluid adhe sion may be increased by maximizing surface tension, minimizing the contact angle, or decreasing fluid viscosity. This is achieved, for example, by changing the surfactant concentration in the binder or by coating the powders with better wetting materials [9]. Upstream grinding min imizes surface roughness that improves the wetting of particulate solid. A higher process temperature reduces fluid viscosity which contributes to a better fluid distribution [10].

In analogy to solid mixing mechanisms, the liquid distribution is affected by the mixing tool induced convective transport as well as by mechanical contact dispersion [11]. The term 'contact dispersion' defines interparticle transfer of liquid due to mechanical particle con tacts. Dry particulate contacts can be calculated on the basis of the Hertz model $[12,13]$ and be simulated numerically with Newton's law of motion (Discrete Element Method). However, the models for DEM simulations of moist cohesive particle systems are still in the early 
stages. Mikami, Kamiya and Horio [14], Willett, Adams, Johnson and Seville [15], Soulie, Cherblanc, El Youssoufi and Saix [16], Butt and Kappl [17], Lian and Seville [18] and Schmelzle and Nirschl [19] devel oped different liquid bridge models, that have in common that the liq uid bridge is stable up to a critical particle separation distance at which the bridge ruptures. Based on the numerical solution of the Young Laplace equation mathematical expressions for the liquid bridge force and rupture distance were derived for mono and polydisperse material and materials with different wetting behaviors. As one of the first, Shi and McCarthy [20] presented a thin film model in which the liquid is distributed evenly on the particle surface. After the bridge rup ture the liquid is redistributed on the contact partners according to a parabolic bridge profile. Mani, Kadau and Herrmann [21] calculated the shear induced liquid transport in undersaturated, monodisperse particulate solids based on the thin film model explained with contin uum mechanics. Mohan, Kloss, Khinast and Radl [11] compare four models describing liquid transport with variable liquid layer thicknesses and flow rates for dynamic bridge filling within a shear field. In all the mentioned works, the assumption is made that the liquid immediately spreads over the entire particle surface. However, this assumption is only justified for extremely well wetting materials with low contact angles. In case of a less hydrophilic material wetted with water, a non ideal liquid film is formed. Depending on the contact angle the size of the droplet and the covered particle surface will be different.

In order to incorporate partial wetting in DEM Simulations, compre hensive model extensions like local liquid storage on the particle surface have to be carried out. Washino, Miyazaki, Tsuji and Tanaka [22] used a discretization method [23] to investigate the intra and inter uniformity of the coating of monodisperse particles in a mixing drum. In this work the same discretization method is used to further develop liquid disper sion in DEM simulations. Partial wetting and the formation of drops are implemented, which should lead to physically improved liquid distribu tions and higher accuracies in experimental validation.

\section{Materials and methods}

\subsection{Mixer and sampling}

Experiments and simulations are carried out in a self made vertical mixer (Fig. 1). The mixing vessel has a diameter of $112 \mathrm{~mm}$ resulting in a maximum filling volume of approximately $0.7 \mathrm{l}$. During experi ments the radial fixed sampling holes (Fig. 1 (left)) are masked and not considered in the simulations. At the bottom of the mixing vessel a three bladed mixing tool operates at different rotational speeds. The experimental validation (Section 4.2) is carried out at $36 \mathrm{rpm}$.

The procedure for statistical evaluation of a mixture requires an adequate sampling method (Fig. 2). This involves a minimum number of particles in the sample, in order to detect a low concentrated additive with a given probability in the sample volume. The minimum number of particles per sample can be estimated [24]. In our application the
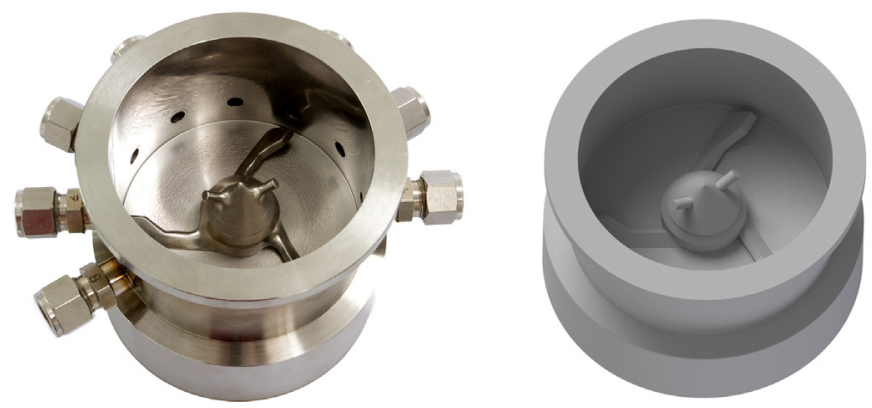

Fig. 1. The vertical mixer (left) and its CAD model (right) used for the experimental validation and the simulation. following applies: with $S=95 \%$ confidence level, the sample composi tion does not deviate more than $y_{s}=7.5 \%$ from the target composition if a particle number $Z$ of at least

$Z \quad\left(\frac{z(S)}{y_{s}}\right)^{2} \frac{1-q_{\text {target }}}{q_{\text {target }}} \quad\left(\frac{1.65}{0.075}\right)^{2} \frac{1-0.5}{0.5} \approx 484$

appears in the sample. The limit factor $z(S)$ depends on the selected con fidence level. Due to the low number of particles $\left(N_{P}=100,000\right)$ a higher probability is not achievable in the context of validation. Additionally the number of samples $N_{S}$ has to be defined. If the empirical variance $s^{2}$ has a probability of $S=95 \%$ to be maximally 1.75 times larger than the true variance $\sigma^{2}$, the required number of samples $N_{S}$ $=16$ can be determined with the Chi square distribution $\chi_{s}^{2}$ :

$\frac{16}{26.3} \frac{f}{\chi_{S}^{2}(S ; f)}>\frac{\sigma^{2}}{s^{2}} \quad \frac{1}{1.75}$.

The values of the Chi square distribution $\chi_{s}^{2}$ and the corresponding degree of freedom $f$ were taken from the textbook by Stieß and Ripperger [25]. In addition to the sample size $Z_{N \text {, } \min }$ and sample number $N_{s}$, the location and frequency of sampling must be defined as well. For a representative determination of the mixing efficiency, the samples have to be distributed randomly over the entire mixing vessel. This guaran tees a holistically assessment of the batch and excludes any systematic signs of segregation which would falsely suggest inferior product qual ities. For this purpose, 16 sampling points were randomly distributed over the entire mixture (Fig. 2a)). The positions were noted and then applied for each sampling time. Depending on the sample position, the rotation of the vessel cap (Fig. 2b)) and the insertion depth of the sampler must be adjusted (Fig. 2c)). With this combination of random and regular sampling, the holistic assessment of the batch is guaranteed in both, experiment and simulation. Due to the high effort, the fre quency of sampling is significantly lower in the experiment but never theless high enough to eliminate systematic sample errors.

The sample compositions are determined by sieving and counting analysis. If the sample composition $q_{i}$ is known, the relative standard de viation $R S D$ can be calculated as a mixing efficiency measure according to:

$\operatorname{RSD} \frac{\left(\frac{1}{N_{s}} \sum_{i 1}^{N_{s}}\left(q_{i}(t)-q_{\text {target }}\right)^{2}\right)^{0.5}}{q_{\text {target }}}$

where $q_{\text {target }}=0.5$ is the predefined number composition. Ideal homo geneity cannot be reached because of random particle motions occuring in solid mixing processes. The best achievable quality of mixing is char acterized by the stochastic mixture $R S D_{z}$, which is function of the sample size:

$\operatorname{RSD}_{Z} \quad q_{\text {target }}\left(1-q_{\text {target }}\right) \frac{1}{Z}$

\subsection{Materials}

Monodisperse glass beads are used as a model product. The filling fraction has a particle radius of approximately $r_{i}=0.92 \mathrm{~mm}$ and is transparent. The additive component is half as large as the filler $\left(r_{j}=\right.$ $0.46 \mathrm{~mm}$ ) and colored red. A different wetting behavior with water arises from the red coating of the individual particles. The static contact angles $\theta$ can be measured by microscope images of a liquid bridge between two particles in contact (Fig. 3 (left)). For this purpose, the toroidal approximation was adapted manually to three images and evaluated using the intersection angles between particle and liquid bridge (Fig. 3 (right)). The red particle shows a contact angle of 

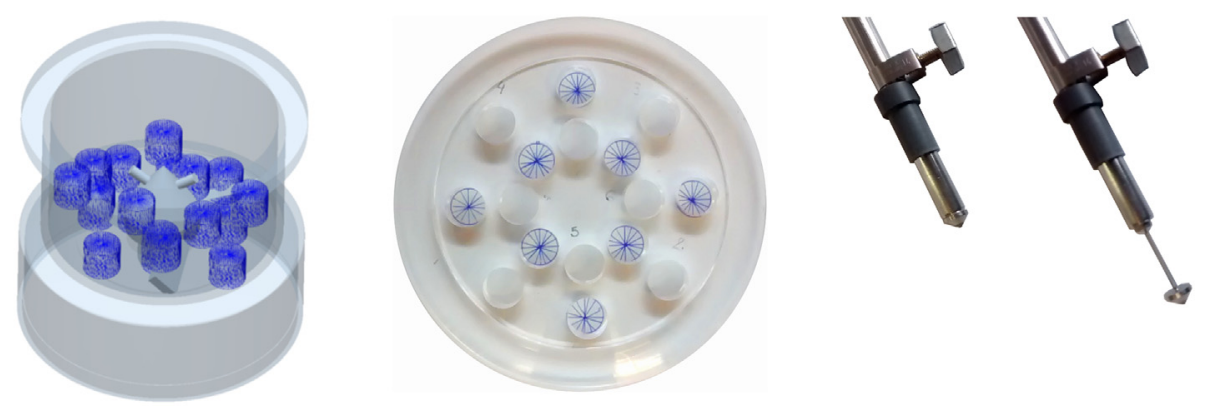

Fig. 2. a): CAD mixer model that illustrates sampling positions. b): A photography of the mixing vessel cap that shows the variable sampling positions in the experiment. c): A photography of the sampler in open and closed position.

$\theta_{j}=67^{\circ}$ and the transparent of $\theta_{i}=12.7^{\circ}$. It should be noted that for the microscope images, only particles of the same particle size are used. This guarantees the liquid bridge to be focused in one plane. In the experi mental and numerical mixing process the red additive is two times smaller than the filler.

\subsection{DEM and liquid bridge model}

The Discrete Element Method is a well developed method to inves tigate the behavior of moving bulk materials and has been applied to a lot of different particle based engineering processes. The method is based on the idea of assuming all objects to be regular spheres [26] with the mass $m_{i}$, that are accelerated according to Newton's transla tional and rotational equations (Eq. (2.5), (2.6)). Therein $I_{i}$ represents the moment of inertia, $\vec{T}_{i j}$ and $\vec{T}_{i j, r}$ are the acting torques resulting from the tangential static and rolling friction. The translational velocity $\vec{u}_{i}$ and rotational velocity $\vec{\omega}_{i}$ of each sphere is obtained by integrating Newton's equation over a short time step $d t$.

$m_{i} \frac{d \vec{u}_{i}}{d t} \quad m_{i} \vec{g}+\sum_{j 1}^{l} \vec{F}_{C n, i j}+\sum_{j 1}^{l} \vec{F}_{B n, i j}$

$I_{i} \frac{d \vec{\omega}_{i}}{d t} \sum_{r=1}^{l} \vec{T}_{i j}+\sum_{j=1}^{l} \vec{T}_{i j, r}$

In moist solids with pendular liquid bridge regime the forces acting on each particle are composed of gravity $m_{i} \vec{g}$, contact forces $\vec{F}_{c n, i j}$ and liquid bridge forces $\vec{F}_{B n, i j}$. The force arising from elastic particle con tacts $\vec{F}_{c n, i j}$ is calculated according to the model by Tsuji, Tanaka and Ishida [13], wherein the particle surface compression is represented by a geometrical overlap. This model is used in the commercial software
EDEM from DEM Solutions by default. When a small amount of liquid is introduced in dry solids, pendular liquid bridges are formed between the particles, which produce attractive liquid bridge forces due to capillary pressure and surface tension. In this work the liquid bridge force model proposed by Mikami, Kamiya and Horio [14] is used, when a moist same particle contact or a moist particle wall contact occurs. An own model [19] is applied in the case of particle particle interaction of different materials.

Liquid bridges with constant volume are stable only up to a certain rupture distance. Consequently, a criterion has to be used that charac terizes the rupture distance, which depends on the particle properties and the volume of the liquid bridge. The same applies to the transfer ratio, which characterizes the amounts of liquid remaining with the particles. In case of same sized particles with the same contact angles, the liquid bridge volume will split 50 to 50 . If there is a difference in particle size, more liquid will remain on the larger particle, unless the wetting properties are significantly better on the smaller particle. Consequently, both rupture distance and transfer ratio are functions of the liquid bridge volume, the contact angles and the particle size ratio. The corresponding equations for calculating the liquid bridge force, the rupture distance and the transfer ratio can be found in $[14,19]$.

Viscous forces were neglected, as we focused on the effect of partial wetting and liquid contact dispersion of water. The capillary number $\mathrm{Ca}$ relates the viscous forces to surface tension forces and is small enough $(\mathrm{Ca}=0.009)$ to legitimate this assumption. The material parameters used to simulate the glass beads (shown in Section 2.2) are listed in Table 1. More detailed information on the contact model used in this work can be found in [19].

\subsection{Micro X ray computer tomography}

Micro X Ray computer tomography was used to analyze the water distribution qualitatively. The used microscope XRadia 520 Versa
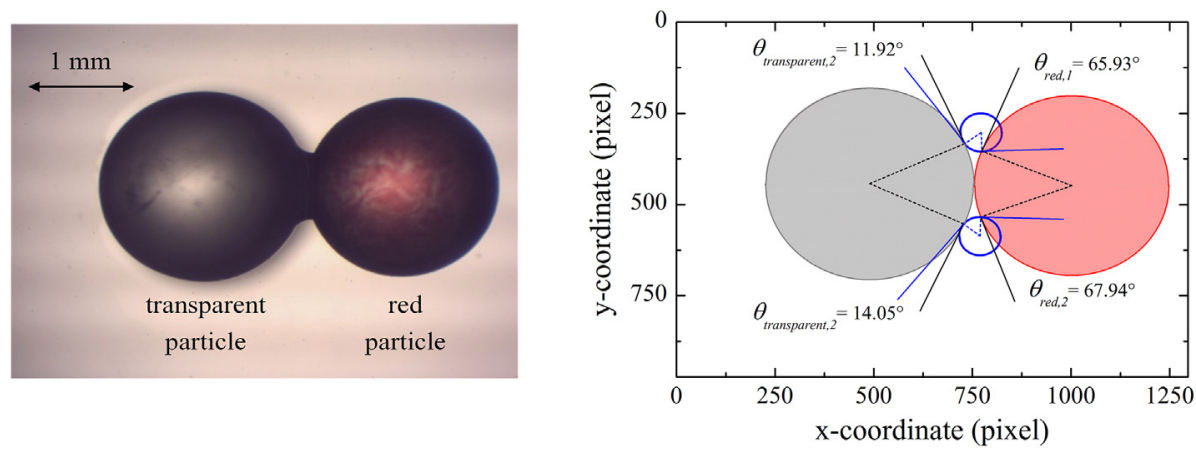

Fig. 3. left: Microscopic image (Leica Wild M3C) of a transparent and color-coated $\mathrm{SiO}_{2}$ particle with a size of $\mathrm{r}_{\mathrm{i}}=0.92 \mathrm{~mm}$. Right: Image analysis of the different static contact angles obtained from the microscopic image on the left. 
Table 1

Material properties used in the simulations.

\begin{tabular}{|c|c|c|}
\hline Properties & Value & Unit \\
\hline filler particle $r_{i}$ & 0.92 & {$[\mathrm{~mm}]$} \\
\hline additive particle $r_{j}$ & 0.46 & {$[\mathrm{~mm}]$} \\
\hline static contact angle of filler particle $\theta_{i}$ & 0 and 12.7 & {$\left[{ }^{\circ}\right]$} \\
\hline static contact angle of additive particle $\theta_{j}$ & $\approx 60 \quad 67$ & {$\left[{ }^{\circ}\right]$} \\
\hline particle density $\rho_{P}$ & 2500 & {$\left[\mathrm{~kg} / \mathrm{m}^{3}\right]$} \\
\hline wall density $\rho_{\text {steel }}$ & 7800 & {$\left[\mathrm{~kg} / \mathrm{m}^{3}\right]$} \\
\hline Shear modulus $G_{p}$ of particles & 216 & {$[\mathrm{MPa}]$} \\
\hline Shear modulus $G_{\text {steel }}$ of steel (mixer) & 8000 & {$[\mathrm{MPa}]$} \\
\hline Coefficient of restitution $e_{p}$, particle to particle & 0.6 & [ ] \\
\hline Coefficient of restitution $e_{\text {steel }}$, particle to wall & 0.7 & [ ] \\
\hline Poisson's ratio of particles $v_{P}$ & 0.25 & [ ] \\
\hline Poisson's ratio of steel $v_{\text {steel }}$ & 0.3 & [ ] \\
\hline Static friction of particles on glass $\mu_{s, p}$ & 0.5 & [ ] \\
\hline Static friction of particles on steel $\mu_{s, ~ s t e e l}$ & 0.32 & [ ] \\
\hline Rolling friction of particles on glass $\mu_{r, p}$ & 0.005 & [ ] \\
\hline Rolling friction of particles on steel $\mu_{r \text {, steel }}$ & 0.02 & [ ] \\
\hline Surface tension of water at $20^{\circ} \mathrm{C}$ & 72.8 & $\mathrm{mN} / \mathrm{m}$ \\
\hline density of water $\rho_{l}$, & 1000 & {$\left[\mathrm{~kg} / \mathrm{m}^{3}\right]$} \\
\hline
\end{tabular}

(Zeiss, Germany) consists of a tungsten $\mathrm{x}$ ray source and a detector unit. The horizontal position of both units can be adjusted to achieve differ ent geometrical magnifications. In between those units is the sample stage, which rotates the sample through $360^{\circ}$. During this rotation typically between 1601 and $2401 \mathrm{x}$ ray images are taken. Afterwards a $3 \mathrm{D}$ representation of the sample is reconstructed from the $\mathrm{x}$ ray images at different angles. Since the likelihood that a material absorbs $\mathrm{X}$ ray quanta depends on its electron density, the final output volume is a grayscale image, with different levels of gray representing different materials or electron densities. It should be noted that other effects like, for instance, beam hardening may also influence the gray levels. Therefore, it is necessary that the contrast between the materials to be separated is large enough.

One sample of filler, additive and water, containing amidotrizoate as a contrast agent was prepared and mixed at its best by hand. The tested sample has $3.2 \%$ mass of water related to the total particle mass and thus a calculated saturation level of approximately $10.2 \%$. The mixture was then filled into a polyimide tube. Sealing the polyimide tube with resin and sticking the tube to the sample holder is necessary to avoid evaporation of water and particle motion during the measurement.

\section{Modelling partial wetting}

\subsection{Fibonacci Lattice method (Fib. Lat.)}

A discretization of the particle surface is introduced in order to realize partial particle wetting in the Discrete Element Method. The Fibonacci Lattice method according to González (2010) allows an almost uniform subdivision of individual Lattice points, in which infor mation can be stored and resolved spatially. Using the inverse of the golden ratio $\Phi^{-1}=0.618$ Swinbank and James Purser [27] show how an odd natural number of Fibonacci Lattice points $N^{\prime}$ is distributed evenly on a spherical surface (Fig. 4). By crossing opposing Fermat spi rals in the golden angle $360^{\circ} \Phi^{-2} \cong 137.5^{\circ}$, the longitudinal $\operatorname{lon}_{k}$ and lat itudinal coordinate $l a t_{k}$ are calculated:

$N^{\prime} \quad 2 N+1$,

lat $_{k} \arcsin \left(\frac{2 k}{2 N+1}\right)$ und $\operatorname{lon}_{k} \quad 2 \pi k \Phi \Phi^{1}$,

where $k$ is an integer from $-N$ to $N$. The discretization method for $N^{\prime}$ $=1001$ Fibonacci Lattice points is shown as an example in Fig. 4. In

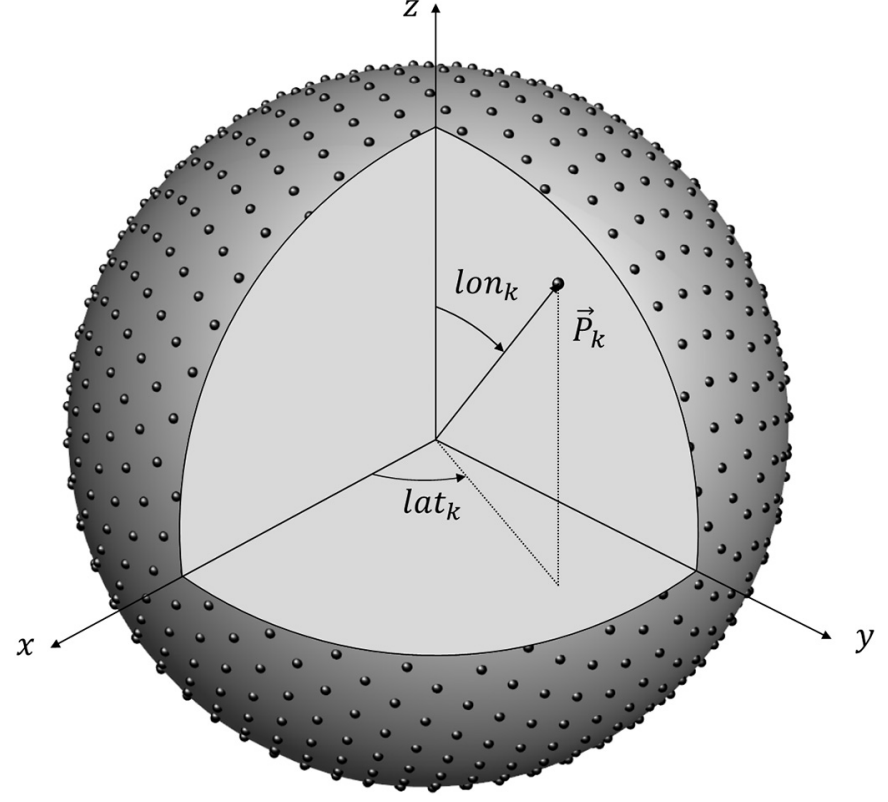

Fig. 4. Particle surface discretized with $\mathrm{N}^{\prime}=1001$ Fibonacci-Lattice points described by longitudinal as well as latitudinal angles.

contrast to the equiangular lattice [28] there are no inhomogeneous accumulations of points at the poles. By definition, the distribution of the points starts at $\vec{P}_{0} \quad\left(\begin{array}{lll}1 & 0 & 0\end{array}\right)^{T}$, which results in an odd total number of points $\vec{P}_{k}$ in spherical coordinates

$\vec{P}_{k} \quad\left(\begin{array}{c}1 \\ \operatorname{lon}_{k} \\ \text { lat }_{k}\end{array}\right)$

Apart from the starting point, each Fibonacci Lattice point has a point symmetric opposite with an opposite sign in the longitudinal and latitudinal angles. This results in a non center symmetric distribu tion, which probably represents the best possible homogeneity in the distances between the individual points [23]. As demonstrated in a Voronoi diagram by Keinert et al. (2015), the distribution of points is particularly incongruent around the starting point $(k=0)$. Applied to the modelling of partial wetting, this means that the area around the pole has the most inhomogeneous distribution of the points and there fore has to be treated critically. However, the homogeneity of the distri bution increases with the number of Fibonacci points. In case of infinitely many points an ideally perfect distribution could be achieved. The discretized points are used to store the amount of liquid locally on the particle surface.

\subsection{Orientation and bridge formation}

If the number of the Fib. Lat. points is defined, the coordinates can be calculated according to Eqs. (3.1) (3.3) and be stored in a look up table. As the particles follow not only the translational but the rotational equa tion of motion (2.6) as well, the initialized Fib. Lat. point coordinates change in each time step. The points rotate around the original coordi nate system, which is described by the Eulerian rotation matrix $\mathbf{Q}$ :

Q BCD. 
D

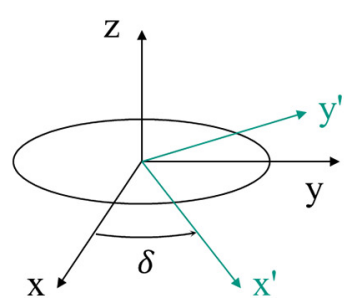

C $\cdot \mathbf{D}$

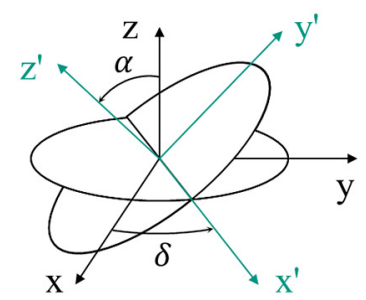

$B \cdot C \cdot D$

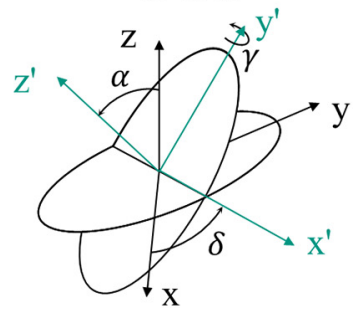

Fig. 5. Euler angles to describe a body-fixed coordinate system.

with the rotation matrices $\boldsymbol{D}, \boldsymbol{C}$

D $\quad\left(\begin{array}{ccc}\cos (\delta) & \sin (\delta) & 0 \\ -\sin (\delta) & \cos (\delta) & 0 \\ 0 & 0 & 1\end{array}\right)$,
C $\quad\left(\begin{array}{ccc}1 & 0 & 0 \\ 0 & \cos (\alpha) & \sin (\alpha) \\ 0 & -\sin (\alpha) & \cos (\alpha)\end{array}\right)$

and $\boldsymbol{B}$ (Fig. 5)

$\boldsymbol{B}\left(\begin{array}{ccc}\cos (\gamma) & \sin (\gamma) & 0 \\ -\sin (\gamma) & \cos (\gamma) & 0 \\ 0 & 0 & 1\end{array}\right)$

The rotation around the $x, y$ and $z$ axes is represented by the three angles $\alpha, \gamma$ and $\delta$. Multiplication with the rotation matrix

$\vec{P}_{k, \text { new }} \quad \boldsymbol{Q} \vec{P}_{k}$

leads to the new coordinates of the point $\vec{P}_{k \text { new }}$. The corresponding point coordinates with respect to the coordinate system of the mixer must be calculated in each time step. A partial wet particle surface is represented, if a liquid quantity is stored in a Fib. Lat. point. A dry surface point is characterized by a value of zero.

When two particles are in physical contact (Fig. 6), a liquid bridge is formed. The liquid bridge volume is calculated according to the spheri cal cap model by tangential line design [20]. The Fib. Lat. points within the blue spherical cap (Fig. 6) will contribute to the liquid bridge volume. For example, the amount of liquid saved in the green point contributes to the liquid bridge, while the amount of liquid saved in red point represents free surface liquid and is still available for an addi tional liquid bridge on the big particle. If the two particles exceed the liquid bridge rupture distance, the liquid will split, redistribute and form a drop adhering on the particle.

\subsection{Drop modelling}

The presented Fib. Lat. discretization offers not only the possibility to save liquid quantities locally, but also to model the drop adhesion. The amount of liquid remaining with the single particle will result from the size and contact angle dependent transfer ratios $[19,20]$. Depending on the wetting properties and the amount of liquid a differ ent shape of drop arises. This subchapter aims to develop an efficient method by which the liquid can be distributed to the Fib. Lat. points in terms of drop representation. For this purpose, the drop is approxi mated by means of a constant radius of curvature $r_{t}$. In analogy to the used liquid bridge models $[14,19]$, the weight of the liquid does not influence the curvature of the drop. If the radius of the particle is $r_{i}$, the radius of the drop is $r_{t}$, and the height of the caps are $h_{i}$ and $h_{t}$, the volume of a spherical drop $v_{t}$ will be obtained by subtrac ting the spherical cap of the particle $v_{k i}$ from the spherical cap of the drop $v_{k t}$ :

$v_{t} \quad v_{k t}-v_{k i} \frac{h_{t}^{2} \pi}{3}\left(3 r_{t}-h_{t}\right)-\frac{h_{i}^{2} \pi}{3}\left(3 r_{i}-h_{i}\right)$.

Both spherical caps are linked by the spherical cap radius $a$ (Fig. 7).

$$
\left.\begin{array}{ll}
h_{i} & r_{i}-\sqrt{ } r_{i}^{2}-a^{2} \\
h_{t} & r_{t}-\sqrt{ } r_{t}^{2}-a^{2}
\end{array}\right\} \hookrightarrow \sqrt{ } r_{i}^{2}-\left(r_{i}-h_{i}\right)^{2} \quad a \quad \sqrt{ } r_{t}^{2}-\left(r_{t}-h_{t}\right)^{2} .
$$

The cap heights and the drop radius vary depending on the wetting angle $\theta_{i}$ and the drop volume. Both, contact angle and drop volume are known in each contact in the DEM simulation. Accordingly, the determi nation of the radius $a$ and the position of the three phase boundary line

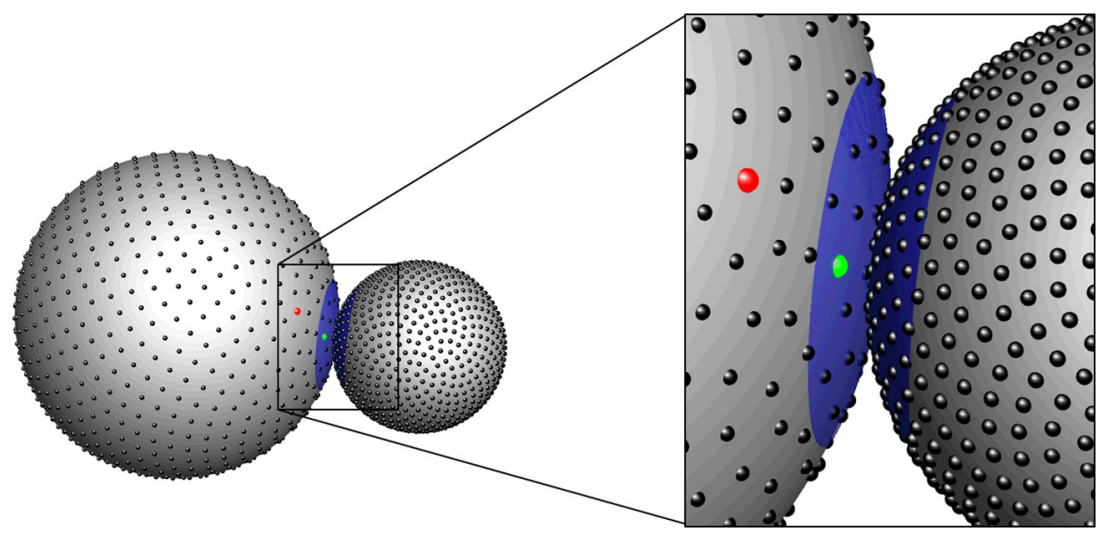

Fig. 6. Spherical cap model proposed by Shi and McCarthy [20]. The liquid within the spherical cap (blue region) will contribute to the liquid bridge volume. The liquid content saved in the green point is part of the liquid bridge volume, while the red point represents free surface liquid. (For interpretation of the references to color in this figure legend, the reader is referred to the web version of this article.) 

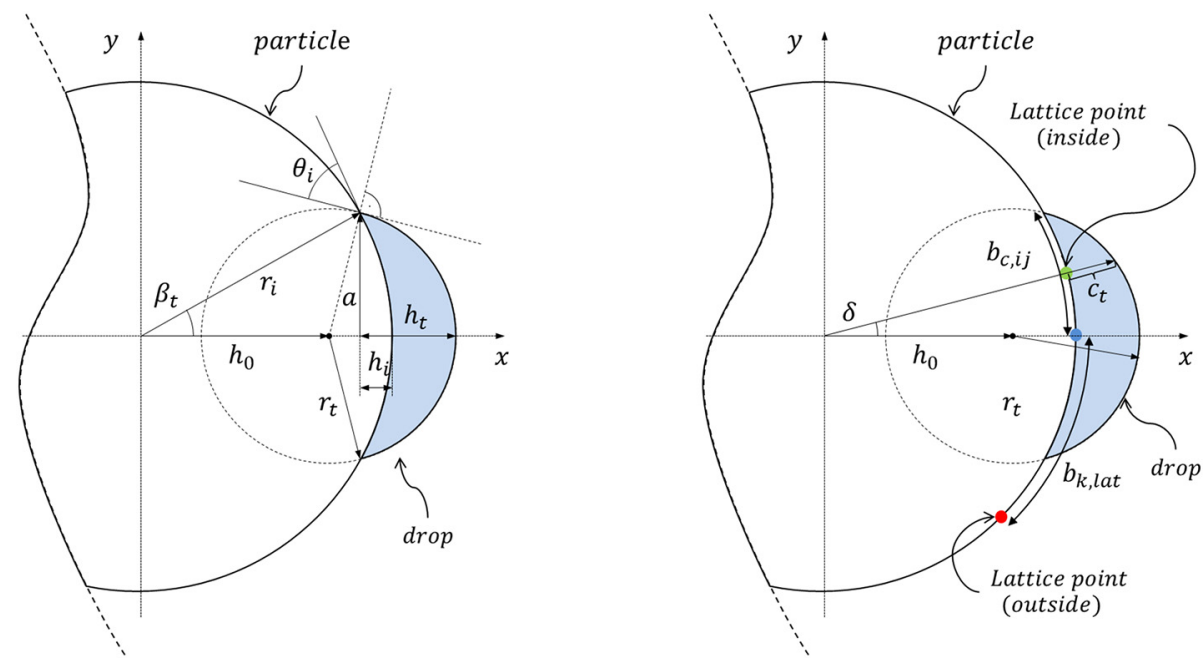

Fig. 7. left: Schematic representation of a drop with the corresponding geometric dimensions. Right: Geometric dimensions that are used to calculate the liquid contents that are saved according to the drop form.

should be done with a simple arithmetic operation. The contact angle is defined by the gradients of the particle and the drop at the three phase contact line.

$\tan \left(\theta_{i}\right)\left|\frac{d y_{i}(x) / d x-d y_{t}(x) / d x}{1+d y_{i}(x) / d x \cdot d y_{t}(x) / d x}\right|$ with $\left\{\begin{array}{cc}y_{i}(x) & \sqrt{r_{i}{ }^{2}-\left(r_{i}+x-h_{i}\right)^{2}} \\ y_{t}(x) & \sqrt{r_{t}}{ }^{2}-\left(r_{t}+x-h_{t}\right)^{2}\end{array}\right.$

Because of the algebraic and trigonometric functions in Eqs. (3.8) (3.10), no analytical solution can be found in order to calculate the dimensionless cap radius $a / r_{i}$ depending on the dimensionless drop vol ume $v_{t} / v_{i}$ :

The numerically determined relationship is shown double logarith mic in Fig. 8 (left). For very small drop volumes, the cap height $h_{i}$ is very little so that the drop adhering to the particle corresponds to the case of flat wall adhesion. This results in a linear increase over a wide range of the dimensionless volume. With larger contact angles, smaller cap radii are observed at a constant volume. With larger drop volumes, the slope decreases progressively to the limit at $\left(a / r_{i}\right)_{\max }=1$. A maxi mum saturation of $13 \%$ is used in the mixing processes (compare chap ter 4), which results in no larger drops than that limit. The bending of the curves in Fig. 8 (left) is due to the fact that the curvature of the par ticle scales cubically with increasing drop volume. Because of the high numerical effort an approximation (3.11) is derived and its mean square error MSE (Eq. (3.12)) is shown in Fig. 8 (right):

$$
\begin{aligned}
& \left(\frac{a}{r_{i}}\right)_{\text {Approx }}\left(1-1 /\left(1+\frac{v_{t}}{v_{i}}\left(\frac{4 \sin ^{3} \theta_{i}}{\left(2-3 \cos \theta_{i}+\cos ^{3} \theta_{i}\right)}\right)\right)\right)^{0.33} . \\
& \text { MSE } \frac{1}{N} \sum_{k i}^{N}\left(\left(\frac{a}{r_{i}}\right)_{\text {Num }}-\left(\frac{a}{r_{i}}\right)_{\text {Approx }}\right)^{2},
\end{aligned}
$$

While the range of very small dimensionless volumes is well approx imated, deviations can be observed for larger volumes. Since the maximum error is $12 \%$ in absolute deviation, the approximation is suffi ciently accurate. It should be noted that this error only affects the shape of the drop a little. The liquid quantities will be unaffected in the simulations.

If the dimensionless spherical cap radius $a$ is known, all other geometric variables in Fig. 7 can be calculated. In the next step the Fib. Lat. points that are shaping the drop have to be determined. As an example, the green point in Fig. 7 (right) is within the wetted particle surface, the red dot is outside. The comparison of the orthodromes be tween the Fib. Lat. point and the contact point (blue point in Fig. 7 (right)) $b_{k}$, lat with the arc length of the wetted surface $b_{c, i j}$ results in the information whether the point participates in the drop or not. This
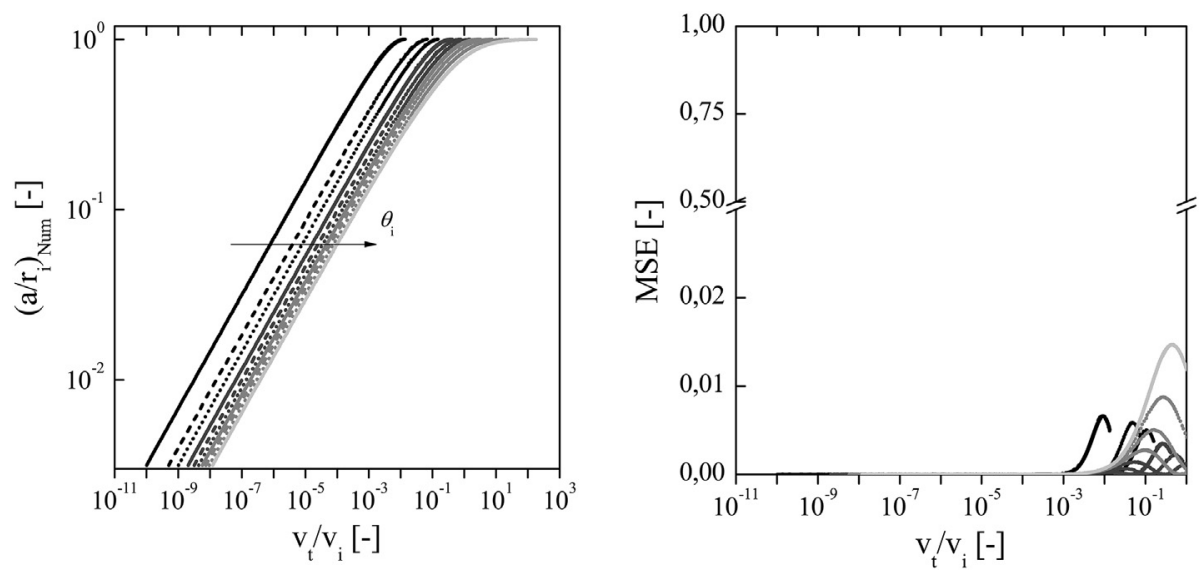

Fig. 8. left: The cap radius a in relation to the particle radius $\mathrm{r}_{\mathrm{i}}$ is a function of the ratio between the drop $\mathrm{v}_{\mathrm{t}}$ and the particle volume $\mathrm{v}_{\mathrm{i}}$, shown for different wetting angles $\theta_{\mathrm{i}}$. Right: Depiction of the mean square error MSE of the numerical solution and the approximation (see Eq. (3.15)). 

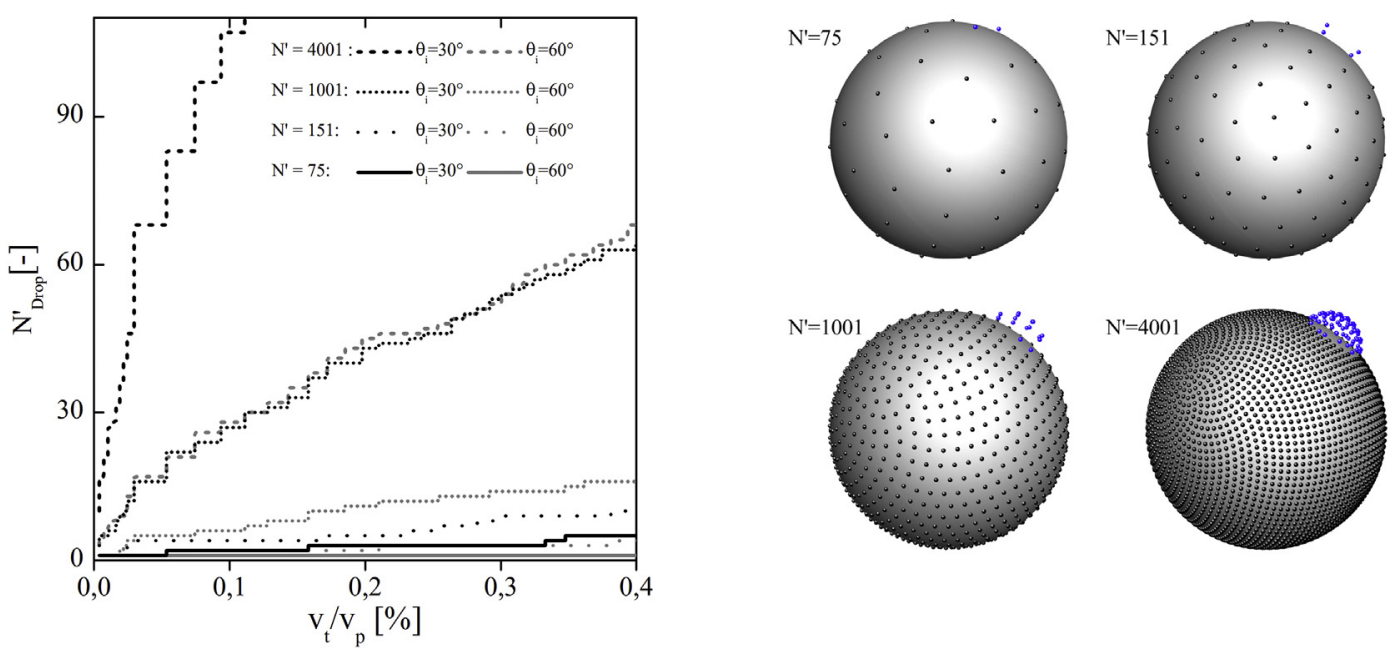

Fig. 9. left: The number of Fib.-Lat. points participating in the drop $\mathrm{N}_{\text {drop }}$ as a function of the amount of liquid $\left(\mathrm{v}_{\mathrm{t}} / \mathrm{v}_{\mathrm{p}}\right)$ and the contact angle of the particle $\theta_{\mathrm{i}}$ at different total Fib.-Lat. numbers $\mathrm{N}^{\prime}$. Right: representation of drop modelling (Eqs. (3.8)-(3.16)) using a contact angle of $\theta_{\mathrm{i}}=60^{\circ}$ and a liquid ratio of $\mathrm{v}_{\mathrm{t}} / \mathrm{v}_{\mathrm{p}}=0.43 \%$ with different numbers of the Fib.-Lat. points $\mathrm{N}^{\prime}$ on the surface. The blue points represent wetted Fib.-Lat. points.

behaviour can be calculated by trigonometric functions and the normal vectors of the Fib. Lat. points $\vec{n}_{f_{k, \text { orent }}}$ and the contact point $\vec{n}_{c p_{i j}}$

$b_{k, \text { lat }} \quad r_{i} \quad \arctan \frac{\left|\vec{n}_{c p_{i j}} \times \vec{n}_{f_{k, \text { orient }}}\right|}{\vec{n}_{c p_{i j}} \vec{n}_{f_{k, \text { rient }}}}$

$\begin{array}{llll}b_{c, i j} & 2 & r_{i} & \arcsin \left(\frac{a}{r_{i}}\right), \quad \quad b_{k, l a t} \leq b_{c, i j} .\end{array}$

Not every Fib. Lat point participating in the drop receives the same amount of liquid. The position of the drop center $h_{0}$ is used to redistrib ute the liquid by means of the drop shape. The larger the distance between the participating Fib. Lat. point and the drop surface $c_{t}(\delta)$, the more liquid will be saved in the lattice point (Fig. 7 (right)). The opening angle $\delta$ between contact point and lattice point varies accord ing to the point position

$$
\frac{c_{t}(\delta)}{r_{i}} \quad \frac{\cos (\delta) h_{0}}{r_{i}}-1 \pm \frac{1}{r_{i}} \sqrt{ } \cos (\delta)^{2} h_{0}^{2}-h_{0}^{2}+r_{t}^{2}
$$

After calculating the distances of the individual points to the particle surface, the drop volume $v_{t}$ is proportionately distributed according to a linear weighting

$v_{\text {Lattice, }, i} \quad v_{t} \frac{c_{t}}{\sum_{l}^{N^{\prime}} c_{t, l}}$,

where the sum over $c_{t}$ describes the total distance of all participating points in the drop. The algorithm of drop formation is explained using an example.

\subsection{Example of drop model}

The drop modelling is exemplified by a drop to particle volume ratio of $v_{t} / v_{p}=0.43 \%$ (Fig. 9). This ratio represents an above average transfer volume within the framework of the numerical investigations. In Fig. 9 (right), a drop that is forming after bridge rupture is illustrated as a function of Fib. Lat. point number $N^{\prime}$. The number of points participating in the drop $N_{\text {drop }}$ increases with a higher number of Fib. Lat. points and thus improves the resolution of the drop simultaneously. As shown in Fig. 9 on the left, this is a function of the contact angle and the drop volume. A better wetting of the particle follows from lower contact angles, resulting in more Fib. Lat. points in the wetted part of the parti cle surface. Physical accuracy is expected to increase with a higher num ber of Fib. Lat. points. As the computational effort rises with the number of Fib. Lat. points enormously, the accuracy and simulation duration are complementary to each other. The number of Fib. Lat. points must be selected individually, depending on the absolute number of particles, the amount of liquid and the tolerable simulation duration.

\section{Results}

As the computation time increases with the number of Fib. Lat. points, it has to be evaluated how the number of points influences the mixing process. Therefore, as a first step, the number of points is discussed in a mixing drum with reduced particle number $N_{P}=2000$. Subsequently, a comparison of the models is shown on the basis of experimentally determined mixing efficiencies and the liquid distribu tion in the vertical mixer.

\subsection{Study on the number of points in a small mixing drum}

The drum rotating horizontally in the thrust regime (Froude Number $=0.01$ ) is considered as an exemplary mixing process. The number of
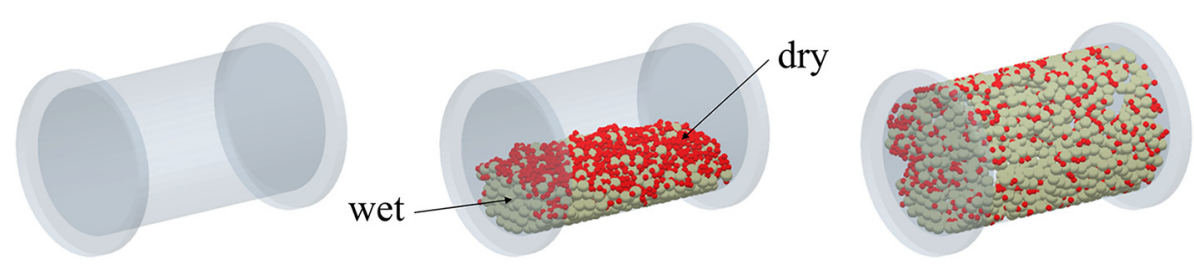

Fig. 10. 3D visualization of an examplary mixing drum with reduced particle number. Left: empty geometry. Middle: initial state after filling. Right: final state after $10 \mathrm{~s}$ of mixing of dry and wet particles. 

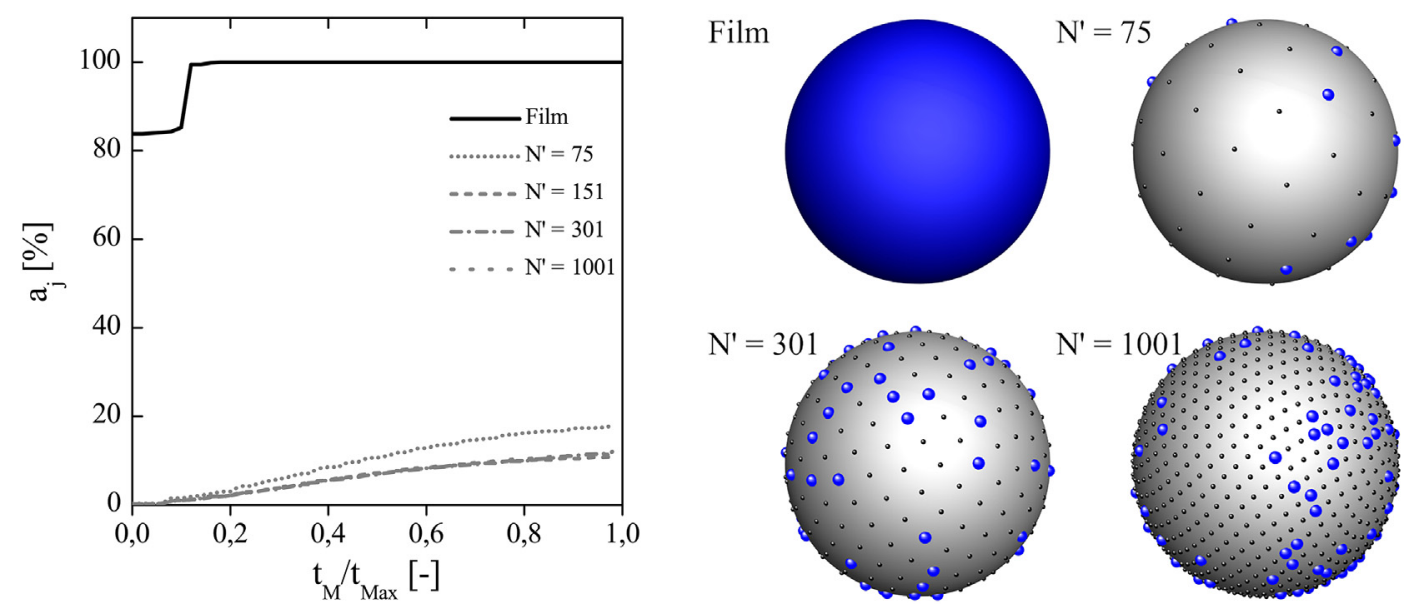

Fig. 11. left: Occupancy rate $a_{j}$ as a function of the dimensionless mixing time $t_{M} / t_{\text {Max }}$. Right: Exemplary illustration of completely (described by the film model) and of partially wetted particles at the end of mixing, depending on the number of Fib.-Lat. points $\mathrm{N}^{\prime}$.

red and gray particles is set to $N_{P, i}$ orj $=1000$. The used material param eters are shown in Table 1. Accordingly, water wets gray (Fig. 10) parti cles much better than red particles. In analogy to the previous investigations [19], the additive is dry in the initial state while each filler particle has allocated a water content of $5 \mathrm{wt}$. \% of the particle mass. In this state (Fig. 10 middle) the total amount of liquid is perfectly dis tributed throughout the filler material. $10 \mathrm{~s}$ of simulation time is consid ered to be sufficient.

The occupancy rate $a_{j}$ is defined as the quotient between the occu pied $N_{j, \text { occupied }}$ and all available Fib. Lat. points $N^{\prime}$ averaged over all fine particles $N_{P, j}$ :

$a_{j} \frac{\sum_{k 1}^{N_{P, j}}\left(N_{j, \text { occupied }}^{\prime}\right)_{k}}{\sum_{k 1}^{N_{P}}\left(N^{\prime}\right)_{k}} \quad \frac{1}{N_{P, j}} \sum_{k 1}^{N_{P, j}}\left(\frac{N_{j, \text { occupied }}^{\prime}}{N^{\prime}}\right)_{k}$.

Because of ideal wetting the occupancy rate of the filler material will remain constant $a_{i}=1$ during mixing. In case of ideal wetting $\left(\theta_{i}=0^{\circ}\right)$ it is a correct approach to apply the film model. Clear differences between the two models are observed, when comparing the occupancy rate of the additive component $a_{j}$ (Fig. 11). In the film model only one point per particle $N^{\prime}=1$ is used to save a liquid quantity which repre sents a thin liquid film covering the whole particle. Hence, the film

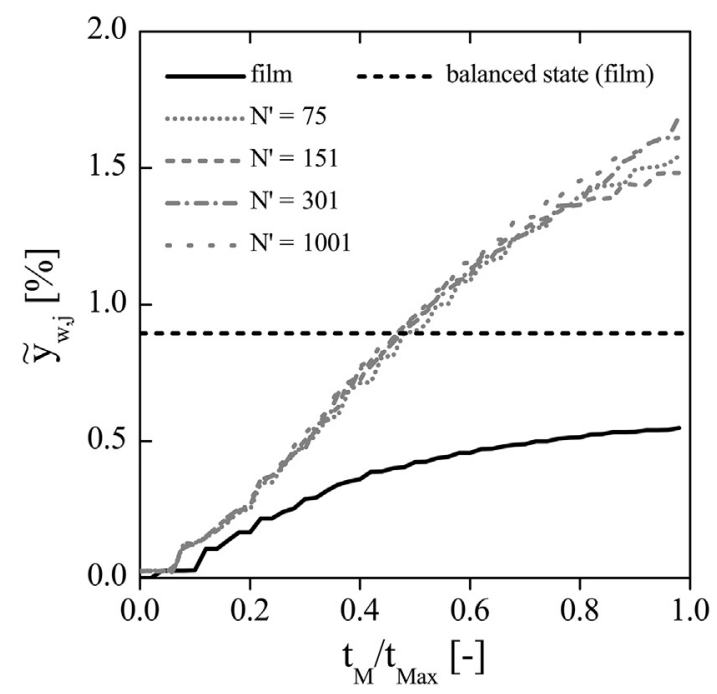

Fig. 12. Mass-specific fraction $y_{w, j}$ that is transferred from the filler to the additive particles as a function of the dimensionless mixing time at a variable number of points $\mathrm{N}$.' model leads to the assumption that particles are either completely dry or completely and ideally wet. As a result of the filling, very few additive particles are initially dry in the film model. With the start of the rotation and a first slippage of the material, the original red dry par ticles suddenly absorb liquid. From this point on (approximately at $t_{M} /$ $t_{\max }=0.1$ ) all particles are wet, indicated by an average occupancy rate of $a_{j}=100 \%$. However liquid transfer is not completed at this time of mixing.

The information on partial particle wetting is given in the new model only. At the end of mixing the majority of the particle surface remains dry. The number of points per particle does not influence the occupancy rate, when it is selected higher than $N^{\prime}=151$. A similar inde pendence of $N^{\prime}$ is observed, when discussing the transferred mass spe cific fraction $y_{w, j}$ :

$y_{w, j} \frac{m_{w, j}}{m_{w, i j}} \quad \frac{m_{w, j}}{m_{w, i}+m_{w, j}}$,

where $m_{w, j}$ is the absolute amount of water on the fine component $j$ and $m_{w, i j}$ is the total amount of water in the mixer. Regardless of the num ber of points, more and more water is transferred to the originally dry particle (Fig. 12 (left)). The film model is aiming for a steady end value while higher mass fractions can be seen in the Fib. Lat. model. Due to low mixing intensity within the drum and high particle wall adhesion, the film model does not reach the balanced state in the selected mixing time. A balanced state is reached when no further liq uid exchange takes place. In the new model a balanced state cannot
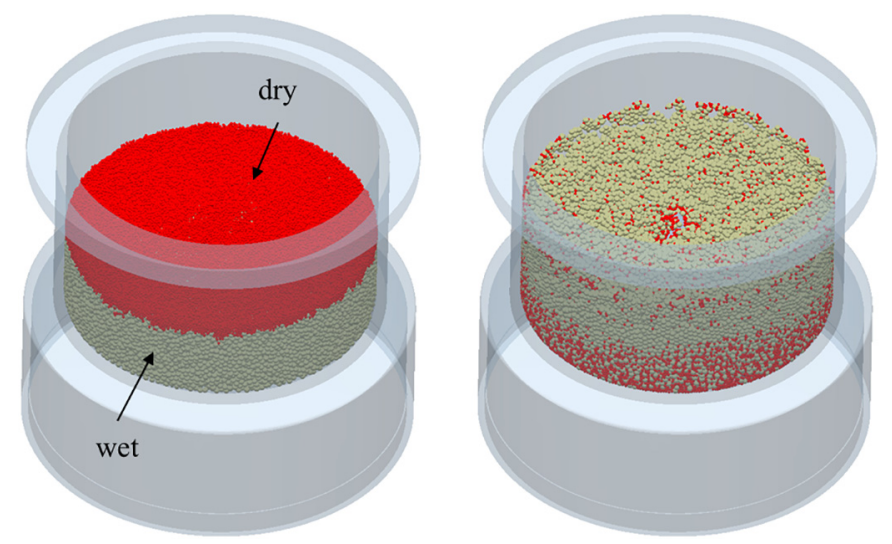

Fig. 13. left: 3D visualization of the mixing vessel with wet and dry particles still seperated at the initial state. Right: 3D visualization of the final state. 

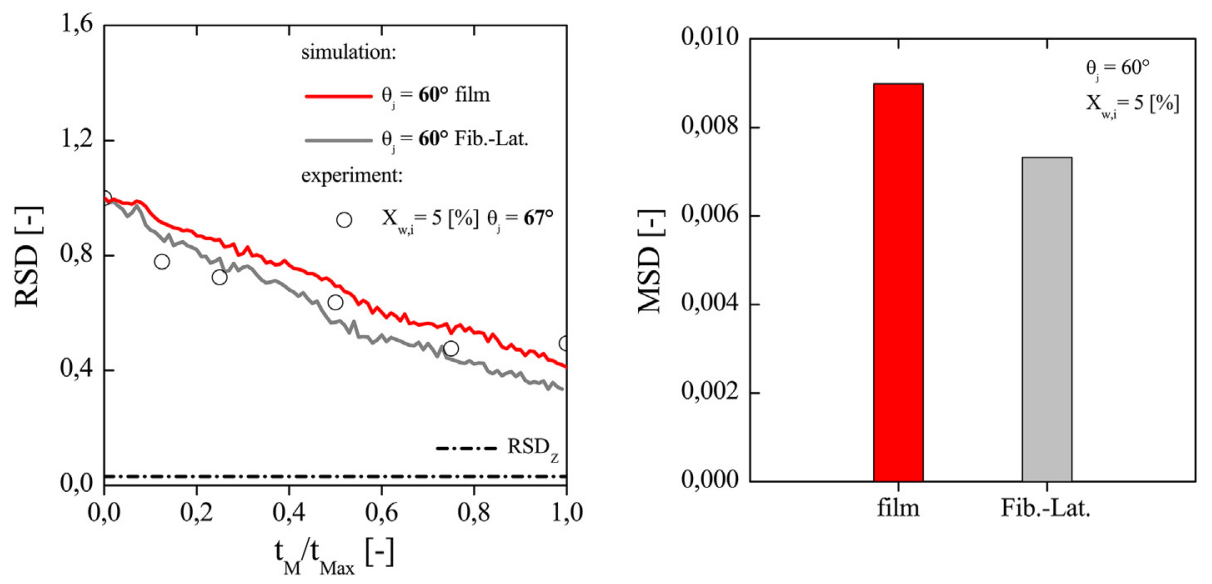

Fig. 14. left: Comparison between film and the Fib.-Lat. model in terms of the mixing efficiencies RSD at $36 \mathrm{rpm}$. Right: Mean square deviation MSD of the models to the experimental data.

be found due to locally distributed liquid content storage. Most of the liquid saved in the points contribute to liquid bridges. Only a few points represent drops in a no particle contact region. This is assessed to be a more realistic state, because the liquid is repeatedly redistributed by particle mixing and contact dispersion.

In summary, the parameter study shows that the occupancy rate and the transferred masses are hardly changed with the number of points. By contrast, the model related differences between complete and partial wetting are significantly larger. Based on these findings, it can be concluded that the model accuracy improves as the number of points increases, but the associated increasing computational effort is not justified. Therefore, a memory point number of $N^{\prime}=75$ is used in the following simulation.

\subsection{Vertical mixer}

\subsubsection{Mixing efficiency}

The validation of the model is performed with the glass beads, water and the mixer shown in chapter 2. In both, simulations and experiment, the loading of the filling material is set to $X_{w, i}=5 \%$ in weight and approximately 50.000 particles were used in each fraction (Fig. 13). The same sample positions were selected in the simulation as well as in the experiment (Fig. 2). The contact angle of the additive component was set to $\theta_{j}=60^{\circ}$ as measured approximately in the experiment (Fig. 3), while perfect wetting on the filler material was assumed in the simulation $\theta_{i}=0^{\circ}$. Here, we accept a small deviation from the experimental data $\left(\theta_{i}=13^{\circ}\right.$ and $\left.\theta_{j}=67^{\circ}\right)$, in order to use the film model on the filler material in the following simulation. It must also be noted that the experimental contact angle measurement is strongly influenced by the manual image evaluation in Fig. 3. Nevertheless, the
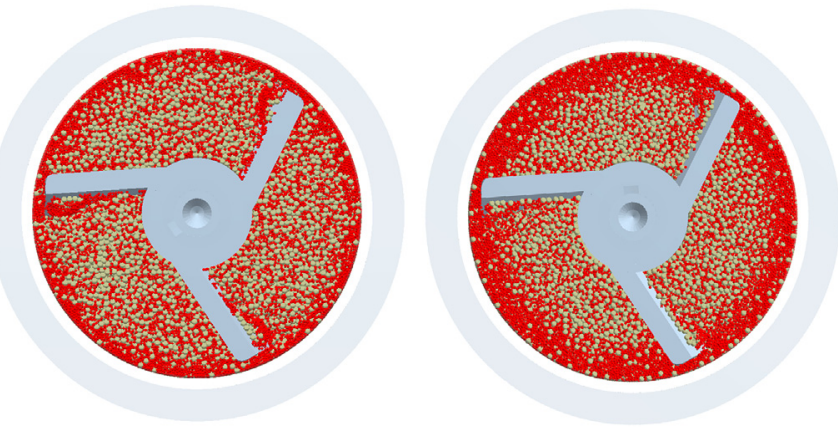

Fig. 15. 3D visualization of the bottom of the vessel at the end of mixing. Left: film model. Right: Fib.-Lat. model. contact angle difference $\Delta \theta=\theta_{j}-\theta_{i}$ is approximately the same in both, simulation and experiment.

Comparing the mixing efficiencies in Fig. 14 (left) one can observe a faster decrease in the RSD of the Fib. Lat. model. This indicates a higher dispersive particle mobility which is due to the higher number of dry particle contacts. In both, film and Fib. Lat. model, the percolating segre gation is not completely repealed, as indicated by the accumulation of red particles at the bottom of the vessel (Fig. 13 (right) and Fig. 15). The dispersive mobility is in turn counteracted by a likewise higher selective transport in the direction of the mixing vessel bottom. The cohesive forces partially improve the homogeneity while percolating segregation is reduced a little. As a result of percolation the stochastic homogeneity $R S D_{Z}$ cannot be achieved at $36 \mathrm{rpm}$ within a simulation time of $t_{M}=20 \mathrm{~s}$.

The calculation of the mean square deviation MSD is used to com pare the two different models with the experimental results.

$\operatorname{MSD} \frac{1}{N} \sum_{k i}^{N}\left(R S D_{\text {simulation }}-R S D_{\text {experiment }}\right)^{2}$,

The mean square deviation MSD reduces from approx. $0.9 \%$ to approx. $0.7 \%$ when implementing the Fib. Lat. model. Consequently the implementation of partial wetting is a physical model improvement in terms of mixing efficiency, but simultaneously causes 100 times larger simulation times.

\subsubsection{Liquid distribution}

The relative density distribution of the liquid (Fig. 16) and the mean particle loading within the samples (Fig. 18) is discussed in addition to the mixing efficiencies. The relative density distribution $q_{r}\left(X_{w, j}\right)$ of the particle loading in the additive fraction is shown at three different times $\left(t_{M} / t_{\max }=0,0.5\right.$ and 1$)$ in Fig. 16. In the beginning most of the particles are dry, which is indicated by $X_{w, j}=0 \%$. Only a few original dry particles absorb some liquid while filling. At a mixing time of $10 \mathrm{~s}$ all particles of the coarse and fine material reach the same final value in the film model (Fig. 16 (left)). In contrast to that a unimodal liquid distribution is reached in the Fib. Lat. model (Fig. 16 (right)).

This represents a methodological improvement, as it is substantiated by the qualitative comparison to Micro X Ray Computer Tomography $(\mu \mathrm{CT})$ recordings (Fig. 17). In analogy to the simulation results, a liquid bridge is formed in almost every contact point. In contrast to the simu lations, however, the particles do not have a homogeneous liquid film on the surface. This means that most of the fluid is bound directly in the bridges and hardly any surface fluid can be detected. On the one hand the three dimensional representation of the entire liquid within the sample shows partially saturated areas, which cannot be 

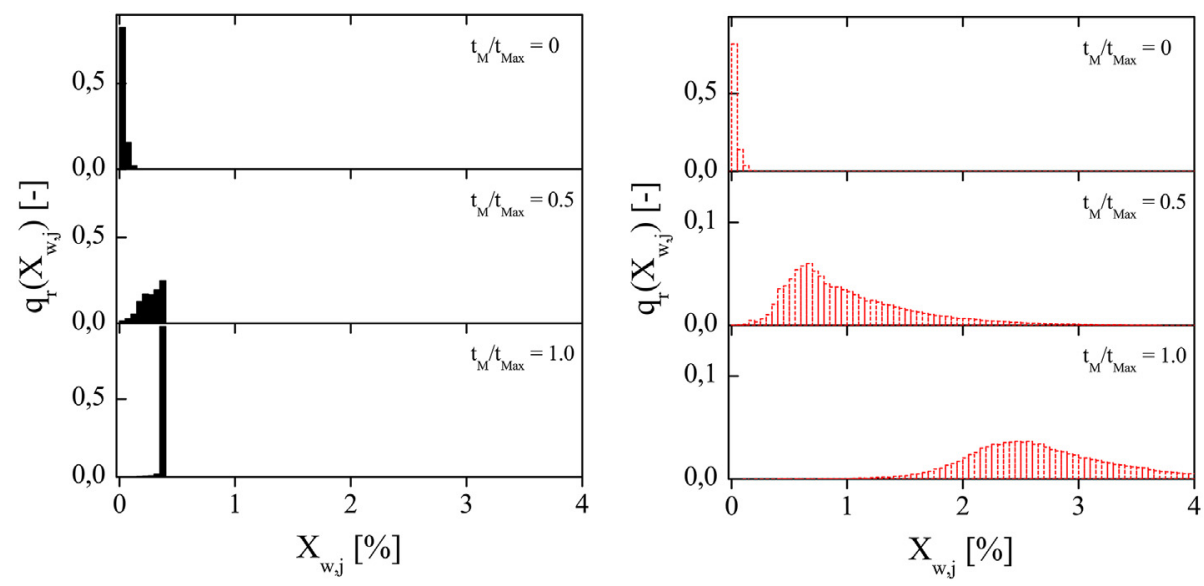

Fig. 16. Relative density distribution $q_{r}\left(X_{w, j}\right)$ of the particle loadings in the additive material at different points in mixing time $t_{M} / t_{\text {max }}$. Left: film model. Right: Fib.-Lat. model.

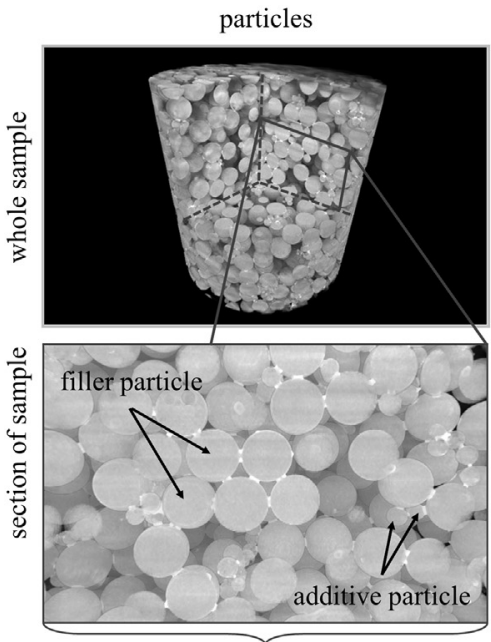

analysis in grayscale
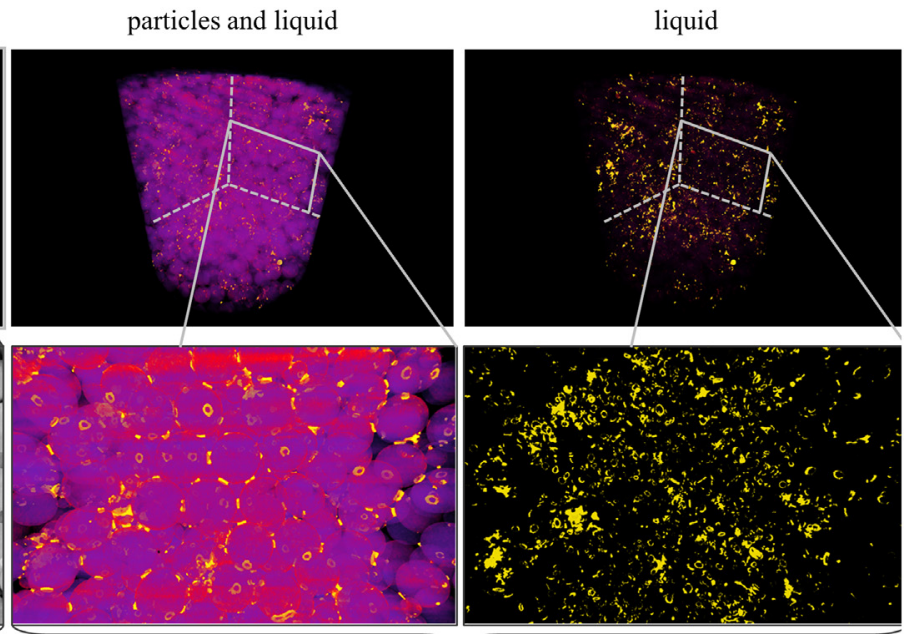

colored analysis

Fig. 17. 3D recordings from ZEISS XRadia Versa 520 ( $\mu \mathrm{CT}$ - micro computer tomography) of a sample. The display of the liquid increases from left to right (yellow color), while particle representation disappears. The entire sample is shown in the upper row, a section with higher resolution is depicted in the lower line. Two different resolutions are displayed to improve the clarity and understanding. (For interpretation of the color in this figure, the reader is referred to the web version of this article.)

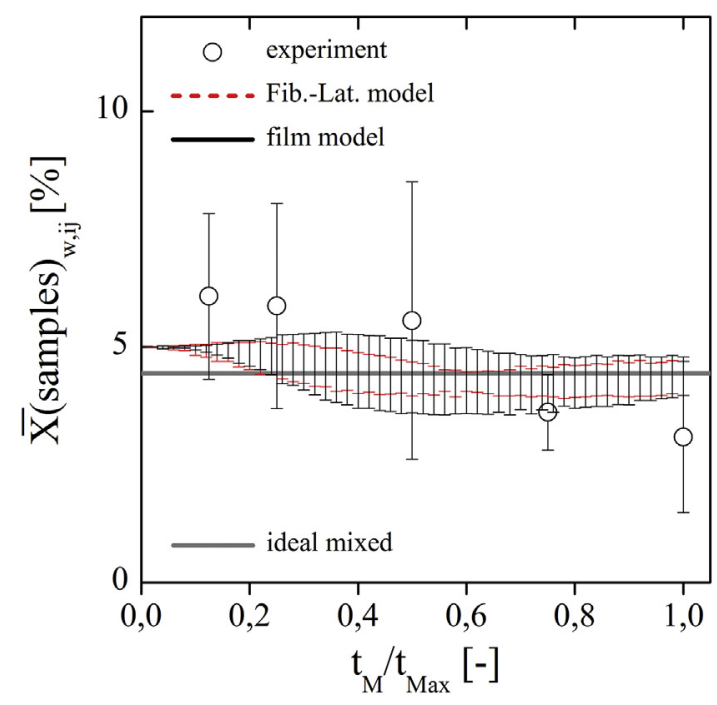

Fig. 18. Averaged particle loading within the samples as a function of the mixing time. The error bars represent the standard deviation of 16 samples. detected by either the film model nor the Fib. Lat. model. Both simula tion models describe the liquid bridge regime. On the other hand, a qualitative spatial liquid distribution is shown in the evaluated sample section. This is reflected much better by the Fib. Lat. than the film model.

In the experimental evaluation of the mixing process it is neces sary to dry the samples before sieving. Therefore it is possible to determine the mass of water in the samples by difference formation. In Fig. 18 the averaged particle load $\bar{X}$ (samples $)_{w, i j}$ is shown as a func tion of the dimensionless mixing time. According to the film an ideal liquid distribution is reached in the samples when the average particle load decreases from $5 \%$ to $4.44 \%$. In both, the experiment and the two simulations this final state is reached. Especially the experimental data have to be discussed critically. The experimentally observed fluctua tion is indicative for a broad liquid distribution in the solids. At the same time, it cannot be quantified how the sampler and the analytical method influence the liquid fluctuation. There is much more particle mass in each sample volume than mass of water. Consequently, a higher error in liquid weighing occurs. As both models do not describe the experimental behavior well, one might assume a percolating effect of water. A local temperature increase additionally occurs as a result of the mechanical energy input and the resulting particle particle 
and particle wall friction, which lead to evaporation of the water. Experimentally, however, the thermal effects at low speed and short mixing time do not seem to have a significant influence. The local accumulation of liquid on the bottom of the mixing vessel bottom may be an effect that is not covered yet in the simulations. For this purpose, further work is planned in order to completely dissolve the liquid phase by means of coupled Discrete Element Method and Volume of Fluid methods.

\section{Conclusion}

In summary, the new method including partial wetting represents a model improvement due to higher validation accuracy. Disadvantages are the computationally intensive costs, so that parameter studies with regard to the number of Fib. Lat. points can only be carried out within an exemplary mixing drum with reduced particle number. Detailed variations of the operating parameters in the pharmaceutical mixer remain open. The partially dry contact points of the additive particles in the new model show a more pronounced percolating segre gation mechanism than in the film model. This observation coincides with the experimental mixing efficiency. The $\mu \mathrm{CT}$ images used to assess liquid distribution qualitatively underpin the improved accuracy of the new model, although dynamic wetting [29] or even fluid flows [30] are not yet considered. For ongoing work, the Fib. Lat. points could be used to implement roughness distribution on the particle surface to represent non smooth spherical particles.

\section{Acknowledgments}

The authors gratefully acknowledge the financial support by the Deutsche Forschungsgemeinschaft (DFG NI 414/25 1).

\section{References}

[1] V. Wenzel, H. Nirschl, Validation of an inline particle probe in a high-shear mixer for particle size determination, Powder Technol. 269 (2015) 178-184.

[2] R. Turton, Challenges in the modeling and prediction of coating of pharmaceutical dosage forms, Powder Technol. 181 (2008) 186-194

[3] G. Toschkoff, S. Just, A. Funke, D. Djuric, K. Knop, P. Kleinebudde, G. Scharrer, J.G Khinast, Spray models for discrete element simulations of particle coating processes, Chem. Eng. Sci. 101 (2013) 603-614.

[4] B. Freireich, R. Kumar, W. Ketterhagen, K. Su, C. Wassgren, J.A. Zeitler, Comparisons of intra-tablet coating variability using DEM simulations, asymptotic limit models, and experiments, Chem. Eng. Sci. 131 (2015) 197-212.

[5] T. Wüstenberg, Cellulose and cellulose derivatives in the food industry: fundamentals and applications, John Wiley \& Sons, 2014.
[6] T. Suzuki, H. Kikuchi, S. Yamamura, K. Terada, K. Yamamoto, The change in characteristics of microcrystalline cellulose during wet granulation using a high-shear mixer, J. Pharm. Pharmacol. 53 (2001) 609-616.

[7] D.M. Parikh, Handbook of pharmaceutical granulation technology, CRC Press, 2016.

[8] K. van den Dries, H. Vromans, Relationship between inhomogeneity phenomena and granule growth mechanisms in a high-shear mixer, Int. J. Pharm. 247 (2002) 167-177.

[9] P. Serno, P. Kleinebudde, K. Knop, Granulieren: Grundlagen, Verfahren, Formulierungen, ECV-Editio-Cantor-Verlag, 2007.

[10] J. Litster, B. Ennis, The science and engineering of granulation processes, Springer Science \& Business Media, 2013.

[11] B. Mohan, C. Kloss, J. Khinast, S. Radl, Regimes of liquid transport through sheared beds of inertial smooth particles, Powder Technol. 264 (2014) 377-395.

[12] H. Hertz, On the contact of elastic solids, Journal für di reine und angewandte Mathematik 92 (1881) 110.

[13] Y. Tsuji, T. Tanaka, T. Ishida, Lagrangian numerical simulation of plug flow of cohesionless particles in a horizontal pipe, Powder Technol. 71 (1992) 239-250.

[14] T. Mikami, H. Kamiya, M. Horio, Numerical simulation of cohesive powder behavior in a fluidized bed, Chem. Eng. Sci. 53 (1998) 1927-1940.

[15] C.D. Willett, M.J. Adams, S.A. Johnson, J.P. Seville, Capillary bridges between two spherical bodies, Langmuir 16 (2000) 9396-9405.

[16] F. Soulie, F. Cherblanc, M.S. El Youssoufi, C. Saix, Influence of liquid bridges on the mechanical behaviour of polydisperse granular materials, Int. J. Numer. Anal. Methods Geomech. 30 (2006) 213-228.

[17] H.-J. Butt, M. Kappl, Normal capillary forces, Adv. Colloid Interf. Sci. 146 (2009) $48-60$.

[18] G. Lian, J. Seville, The capillary bridge between two spheres: New closed-form equations in a two century old problem, Adv. Colloid Interf. Sci. 227 (2016) 53-62.

[19] S. Schmelzle, H. Nirschl, DEM simulations: mixing of dry and wet granular material with different contact angles, Granul. Matter 20 (2018).

[20] D. Shi, J.J. McCarthy, Numerical simulation of liquid transfer between particles, Powder Technol. 184 (2008) 64-75.

[21] R. Mani, D. Kadau, H.J. Herrmann, Liquid migration in sheared unsaturated granular media, Granul. Matter 15 (2013) 447-454

[22] K. Washino, K. Miyazaki, T. Tsuji, T. Tanaka, A new contact liquid dispersion model for discrete particle simulation, Chem. Eng. Res. Des. 110 (2016) 123-130.

[23] Á. González, Measurement of areas on a sphere using Fibonacci and latitude-longitude lattices, Math. Geosci. 42 (2010) 49-64.

[24] K. Sommer, Sampling of powders and bulk materials, Springer-Verlag, Berlin, Heidelberg, 1986.

[25] M. Stieß, S. Ripperger, Mechanische Verfahrenstechnik-Partikeltechnologie, Springer-Verlag, 20091.

[26] P.A. Cundall, O.D. Strack, A discrete numerical model for granular assemblies, Geotechnique 29 (1979) 47-65.

[27] R. Swinbank, R. James Purser, Fibonacci grids: A novel approach to global modelling, O. J. R. Meteorol. Soc. 132 (2006) 1769-1793.

[28] M.J. Gregory, A.J. Kimerling, D. White, K. Sahr, A comparison of intercell metrics on discrete global grid systems, Comput. Environ. Urban. Syst. 32 (2008) 188-203.

[29] R. Patel, D. Wang, C. Zhu, Modeling of Liquid Bridge and Surface Wetting on Two Rigid Spherical Particles, ASME 2010 3rd Joint US-European Fluids Engineering Summer Meeting collocated with 8th International Conference on Nanochannels, Microchannels, and Minichannels, American Society of Mechanical Engineers 2010, pp. 397-401.

[30] D. Jain, N.G. Deen, J.A.M. Kuipers, S. Antonyuk, S. Heinrich, Direct numerica'l simulation of particle impact on thin liquid films using a combined volume of fluid and immersed boundary method, Chem. Eng. Sci. 69 (2012) 530-540. 
Karlsruher Institut für Technologie

\section{Repository KITopen}

Dies ist ein Postprint/begutachtetes Manuskript.

Empfohlene Zitierung:

Schmelzle, S.; Asylbekov, E.; Radel, B.; Nirschl, H.

Modelling of partially wet particles in DEM simulations of a solid mixing process.

2018. Powder technology, 338.

doi:10.5445//R/1000084687

Zitierung der Originalveröffentlichung:

Schmelzle, S.; Asylbekov, E.; Radel, B.; Nirschl, H.

Modelling of partially wet particles in DEM simulations of a solid mixing process.

2018. Powder technology, 338, 354-364.

doi:10.1016/j.powtec.2018.07.028 